\title{
Adeno-assoziierte Virus-Vektoren zur Hautgentherapie
}

M. Braun-Falco

\author{
Adeno-Associated Virus Vectors for Cutaneous Gene Therapy
}

\section{Zusammenfassung}

Die Entwicklung sicherer und effizienter Gentransfer-Vektoren ist entscheidend für eine erfolgreiche Gentherapie. Ein viel versprechendes Vektor-System, welches diese Fähigkeiten besitzen könnte, basiert auf rekombinanten adeno-assoziierten Virus Typ 2 (rAAV-2). AAV-2 ist ein humanes apathogenes Mitglied der Parvovirus-Familie. Die Vorteile von rAAV-2 als Vektor liegen im breiten Tropismus, was eine effiziente Transduktion einer Vielzahl von Zelltypen erlaubt, im Potential zur Langzeitgenexpression und spezifischen Integration sowie in der niedrigen Immunogenität. Obwohl bereits klinische Studien mit rAAV-2-Vektoren durchgeführt werden, ist rAAV-2 als Vektor zur Hautgentherapie wenig untersucht. Diese Übersicht fasst die soweit bekannten Erkenntnisse über die Anwendung von rAAV-2-Vektoren am Organ Haut zusammen. Bisher scheinen sowohl die Förderung der Wundheilung als auch die Rekonstitutionstherapie von Genodermatosen mögliche Einsatzgebiete für rAAV-2-Vektoren im Bereich Hautgentherapie zu sein.

\section{Abstract}

The development of safe and efficient gene transfer vectors is crucial for the success of gene therapy. A promising vector system, which might meet these requirements, is based on recombinant adeno-associated virus type 2 ( $\mathrm{rAAV}-2$ ). AAV-2 is a human, apathogenic member of the parvovirus family. The advantages of rAAV-2 as a vector are the broad tropism allowing the efficient transduction of a variety of cell types, the potential to achieve long-term gene expression and to integrate site specifically as well as the low immunogenicity. Although several clinical trials using rAAV-2 vectors are already ongoing, only little is known about the use of $\mathrm{rAAV}-2$ as a vector for cutaneous gene therapy. This review summarizes the few results obtained by the application of rAAV-2 vectors on the skin organ. So far it seems that the stimulation of wound healing and the reconstitution therapy of genodermatoses might be possible areas for the use of rAAV-2 vectors in the field of cutaneous gene therapy.
Die Haut stellt als Zielorgan des Gentransfers ein attraktives Gewebe zur Entwicklung neuartiger Behandlungsmöglichkeiten für eine große Anzahl unterschiedlicher Erkrankungen dar. Hierzu zählt die genetische Immunisierung gegen Krebs oder Infektionskrankheiten, die Förderung der Wundheilung sowie die kausale Behandlung monogen vererbter Genodermatosen als auch systemischer Proteinmangelerkrankungen wie Hämophilie [1 - 4]. Darüber hinaus bietet die Haut gerade im Vergleich zu anderen häufig zum Gentransfer avisierten Organen wie Leber,
Blutzellen und Muskel den Vorteil der leichten Erreichbarkeit und Einsehbarkeit, was eine unproblematische Entnahme von Hautzellen erlaubt, die einmalige Situation der ständigen Kontrollierbarkeit gentherapeutisch veränderten Gewebes und im Falle von Nebenwirkungen die Option einer schnellen und gezielten Exzision des betroffenen Hautareals.

In Abhängigkeit von der Erkrankung, die durch genetische Modulation behandelt werden soll, muss die Applikation einer Gen-

Institutsangaben

Klinik und Poliklinik für Dermatologie und Allergologie am Biederstein, Technische Universität München

Korrespondenzadresse

Dr. Markus Braun-Falco · Klinik und Poliklinik für Dermatologie und Allergologie am Biederstein, Technische Universität München · Biedersteinerstraße 29·80802 München·mbf@lrz.tum.de

Bibliografie

Akt Dermatol 2004; 30: 554-558 @ C Georg Thieme Verlag KG Stuttgart · New York

DOI 10.1055/s-2004-826132 · ISSN 0340-2541 
fähre (Vektor) bestimmte Anforderungen erfüllen, beispielsweise bezüglich der Effizienz des Gentransfers, der gewünschten Dauer der Genexpression, der Spezifität des zu transfizierenden Zelltyps und vor allem der Sicherheit.

Da die „perfekte Genfähre“ bisher noch nicht existiert, ist die Entwicklung eines möglichst effizienten und sicheren Vektors nach wie vor eine der größten Herausforderungen und Aufgaben auf dem Gebiet der Gentherapie.

Eines der viel versprechenden Vektorsysteme, welches bereits in ersten humanen Studien zur Behandlung von Hämophilie und Zystischer Fibrose zum Einsatz kam, basiert auf dem rekombinanten adeno-assoziierten Virus Typ 2 (rAAV-2) [5]. Die Vorteile von rAAV-2 als Vektor liegen unter anderem im breiten Tropismus, was eine effiziente Transduktion einer Vielzahl unterschiedlicher Zelltypen erlaubt, im Potenzial zur Langzeitgenexpression und spezifischen Integration sowie in der niedrigen Immunogenität.

\section{Vom Virus zum Vektor}

Wildtyp-AAV-2 wurde Mitte der 60er-Jahre als Verunreinigung bei der Laborherstellung von Adenoviren entdeckt. AAV-2 sind einzelsträngige, hüllenlose DNS-Viren aus der Familie der Parvoviridae [5-8]. Da sie natürlich Replikations-defizient sind, gehören sie dem Genus Dependovirus an. Zur AAV-2-Replikation bedarf es in der Wirtszelle zusätzlicher viraler Kofaktoren, die beispielsweise von Adenoviren oder Herpesviren bereit gestellt werden. In Abwesenheit dieser Helferfunktion integriert Wildtyp-AAV-2 in bis zu 70\% an einer spezifischen Stelle (AAV-S1) in Chromosom 19q13.3-qter. Die Integration von Therapiegenen an dieser Stelle wird als ungefährlich angesehen, was eine wünschenswerte Voraussetzung für einen Langzeitgentransfer darstellt. Als humaner Virus, dessen primärer Wirt der Mensch ist, scheint AAV als Vektor zur Gentherapie sehr geeignet, da bisher keine Erkrankungen bekannt geworden sind, die bei einer Seropositivität in der erwachsenen Bevölkerung bis zu $70 \%$ auf eine Infektion mit AAV-2 zurückzuführen sind. Neben AAV-2 sind sieben weitere Serotypen bekannt [8].

Das Genom von AAV-2 besteht aus 4680 Nukleotiden, die zwei offene Leserahmen beinhalten, welche für die Replikations(Rep) und Kapsidproteine (Cap) kodieren. Flankiert werden sie zu beiden Seiten von invertierten terminalen Repetitionen (ITR). Die ITRs sind die einzigen in cis notwendigen Virus-eigenen DNSSequenzen, die zur Herstellung rekombinanter Vektorpartikel notwendig sind und erhalten werden müssen. Die gesamten kodierenden Virussequenzen (Rep und Cap) können durch eine Expressionskassette ersetzt werden, in die ein beliebiges Therapiegen eingebaut werden kann. Zur Herstellung von rekombinanten Vektorpartikeln werden Rep und Cap und die darüber hinaus notwendigen adenoviralen Kofaktoren in trans zur Verfügung gestellt [9]. Damit eine effiziente Herstellung von rekombinanten Vektorpartikeln gewährleistet ist, darf das Therapiegen mit allen notwendigen regulatorischen Sequenzen wie Promotor und Poly-A-Bereich zusätzlich beider ITRs die Länge des Wildtypvirus nicht wesentlich überschreiten [10]. Auf diese Weise können Vektor-Lysate mit Titern > $10^{10}$ infektiösen Partikeln/ml hergestellt werden [9].

\section{Allgemeine Aspekte des rAAV-2 vermittelten} Gentransfers in Keratinozyten

Da Keratinozyten eine wesentliche Rolle in der Pathophysiologie von zahlreichen Hauterkrankungen spielen, insbesondere bei unterschiedlichen Genodermatosen, Hautkrebs oder der Wundheilung, kommt dem Gentransfer in Keratinozyten eine besondere Bedeutung zu.

In einer von der Berliner Dermatologischen Gesellschaft mit ihrem Forschungsförderpreis 2000 ausgezeichneten Arbeit wurde erstmals aufgezeigt, dass rAAV-2-Vektoren einen effizienten Gentransfer in humanen Keratinozyten in vitro grundsätzlich ermöglichen [11]. An einem Schweinemodell konnte dies in vivo von Hengge und Mirmohammadsadegh bestätigt werden, wo sich nach intradermaler Injektion von rAAV-2-Partikeln eine Expression des Transgens in der Epidermis und in Haarfollikelzellen bis zu 6 Wochen nachweisen ließ [12]. Außer Keratinozyten ließen sich in der Haut noch Zellen melanozytären Ursprungs effizient transduzieren [13].

In der Folge fiel auf, dass die relative Permissivität von Keratinozyten für rAAV-2 im Vergleich zu den sehr stark rAAV-2-permissiven HeLa-Zellen deutlich erniedrigt ist. Dies zeigte sich vor allem nach Verwendung von Vektor-Lysaten, die nach einer neu entwickelten, vollständig Adenovirus-freien rAAV-2-Herstellungsmethode aufbereitet waren und den bekannten Effekt der Transduktionserhöhung durch kontaminierende Adenoviren vermissen ließen [9]. Aus dieser Problematik ergaben sich die Fragen, welche Faktoren Einfluss auf die rAAV-2-Transduktion von Keratinozyten nehmen und die relativ niedrige Permissivität erklären könnten bzw. durch welche Faktoren sich die Transduktionseffizienz bei Keratinozyten erhöhen ließe.

Betrachtet man den Infektionsweg von AAV-2 [6], so steht an erster Stelle die Virusaufnahme in die Zelle. Bei AAV-2 ist diese Rezeptor-vermittelt. Da Keratinozyten sowohl die notwendigen Primärrezeptoren (Heparansulfatproteoglykane) als auch Korezeptoren (FGF-R, Integrin) auf ihrer Oberfläche exprimieren, erscheint es primär unwahrscheinlich, dass die Zellaufnahme gestört ist. Nach Rezeptor-vermittelter Endozytose werden die Viruspartikel innerhalb des Zytoplasmas in Endosomen zum Nukleus transportiert, wo sie in den Perinuklearraum freigegeben werden. Bei Keratinozyten zeigte sich wie auch bei anderen Zellarten, dass Virione auf diesem intrazellulären Transportweg durch Proteasomen abgebaut werden bzw. durch Inhibition der Proteasomenaktivität eine deutliche zumindest temporäre Erhöhung der Transduktionseffizienz zu erzielen ist [14-16]. Nach der Aufnahme in den Nukleus muss die einsträngige Virus- bzw. Vektor-DNS in eine doppelsträngige transskriptionsaktive DNS synthetisiert werden, damit eine Genexpression zustande kommt. Eine entscheidende Rolle spielt dabei der Phosphorylierungszustand eines zellulären Proteins namens Einzelstrang D-Sequenz-Bindungsprotein (ssD-BP), der wiederum durch die Tyrosinphosphokinase des epidermalen Wachstumsfaktor-Rezeptors beeinflusst (EGF-R TK) wird [17]. Da EGF für das Wachs- 
tum von Keratinozyten wichtig ist, war zu erwarten, dass EGF-R TK bei Keratinozyten die Transduktion beeinflusst. Dementsprechend konnte eine mehrere Wochen anhaltende Erhöhung der Genexpression durch Blockierung von EGF-R TK erwirkt werden [16].

Da sowohl Proteasomen-Inhibitoren als auch EGF-R TK-Inhibitoren als Medikamente in klinischer Anwendung sind, könnten sie künftig zu einer Optimierung der rAAV-2-Transduktion von Keratinozyten herangezogen werden.

Anwendungsbereiche von rAAV-2-Vektoren in der Hautgentherapie

Die Förderung der Wundheilung durch Wachstumsfaktoren erfährt zunehmendes Interesse innerhalb der letzten Jahre. Nachdem man die regulatorischen Funktionen von mehreren Wachstumsfaktoren in den unterschiedlichen Wundheilungsstadien erkannt hat, wurden die Einzelsubstanzen auf ihr Potenzial zur Förderung der Wundheilung ausgetestet. Dies führte beispielsweise zur Markteinführung von topisch applizierbarem rekombinanten Blutplättchen-abstammenden Wachstumsfaktor PDGF (Becaplermin-Gel). Der Nachteil der topischen Applikation von Wachstumsfaktoren ist jedoch deren kurze Halbwertszeit, der schnelle Abbau im Wundsekret und deren schlechte Bioverfügbarkeit aus den verwendeten Grundlagen. Eine Möglichkeit, die Dauer und Effizienz zu erhöhen, wäre der direkte Gentransfer von Wachstumsfaktoren in das Wundbett [2]. In diesem Zusammenhang wurden rAAV-2-Vektoren bereits an unterschiedlichen Tiermodellen mit Erfolg eingesetzt. So führte der Gentransfer von vaskulärem endothelialen Wachstumsfaktor VEGF zur Stimulation der Angiogenese und zu einer verkürzten Heilungsphase bei Inzisions-, Exzisions- und Verbrennungswunden [18-20].

Ein anderes Anwendungsgebiet könnten Erbkrankheiten sein. Da rAAV-2-Vektoren zumindest theoretisch das Potenzial zur Integration des Transgens in die chromosomale DNS der Zielzelle aufweisen und in einigen Tiermodellen bereits einen Langzeitgentransfer von mehr als einem Jahr vermittelten, scheinen sie zur Therapie von Genodermatosen, bei denen eine langfristige Korrektur erwünscht ist, geeignet [5-8]. Für einen Langzeitgentransfer an der Haut ist zusätzlich entscheidend, dass Keratinozytenstammzellen stabil transduziert werden können. Nur so ist gewährleistet, dass das Therapiegen im Kompartiment der ständigen Epidermiserneuerung verankert bleibt und nicht innerhalb einer epidermalen Regenerationsphase von ca. 28 Tagen verloren geht [4]. Obwohl Keratinozytenstammzellen auf unterschiedliche Weise charakterisiert werden können, existiert keine definitive Nachweismethode. Eine der Möglichkeiten besteht in der Überprüfung der klonalen Wachstumseigenschaften von Keratinozyten in vitro [21]. Abb. 1 gibt ein Beispiel eines aus transfizierten Keratinozyten herangewachsenen Klons. Hieraus lässt sich mutmaßen, dass rAAV-2-Vektoren Keratinozyten mit Stammzell-artigem Wachstumsverhalten transduzieren können. Eine letztlich zuverlässige Aussage wird jedoch erst nach Transplantation modifizierter Stammzellen in vivo möglich sein.

Welche Genodermatosen kommen für eine Gentherapie mittels rAAV-2-Vektoren in Betracht?
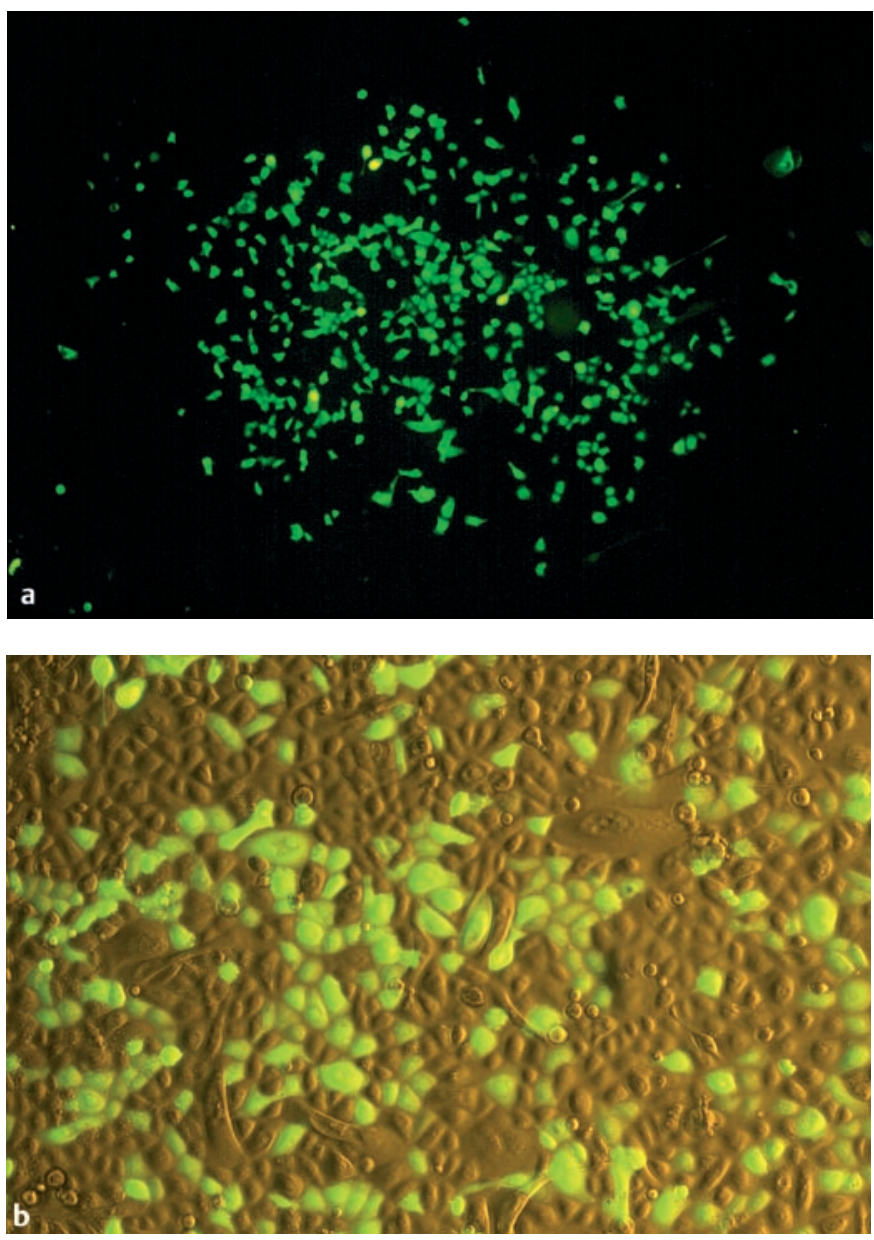

Abb. 1 Klonartig herangewachsenes Zellkonglomerat aus Keratinozyten, in die 11 Tage vorher mit rAAV-2-Vektoren die cDNS für grün fluoreszierendes Protein eingebracht wurde (a Überblick mit 100 x, b Detail mit $400 \mathrm{x}$ ).

Grundsätzlich jede, deren Gendefekt bekannt ist und deren funktionelle DNS die Verpackungskapazität des Vektors nicht überschreitet. Berechnet man für regulatorische Elemente ca. 2000 Basenpaare, ließen sich Therapiegene bis zu einer Größe von ca. 2500 Basenpaaren in den Vektor einbauen. Eine Auswahl von Genodermatosen, die diese Voraussetzung erfüllen, ist in Tab. 1 gegeben.

Die eigene Arbeit hat sich auf das Sjögren-Larsson Syndrom (SLS) konzentriert. SLS ist eine autosomal-rezessiv vererbte, neurokutane Krankheit, die klinisch durch die Trias kongenitale Ichthyose, mentale Retardierung und Spastik bis hin zur Di- oder Tetraplegie charakterisiert ist [22]. Der dem Krankheitsbild zugrunde liegende Defekt befindet sich im Gen für Fettaldehyddehydrogenase (FALDH) [23]. FALDH ist ein Nicotinamid-Adenin-Dinukleotid (NAD)-abhängiges Enzym, welches als Bestandteil des Fettalkohol:NAD+ Oxidoreduktase-Komplexes (FAO) für die Oxidation präferentiell langkettiger aliphatischer Aldehyde zu Fettsäuren notwendig ist [23]. Bei SLS liegt ein Mangel an FALDH mit einer Enzymaktivität unter 10\% der Norm vor. Die Behandlung ist bisher rein symptomatisch. Lediglich eine Therapie mit Leukotrien B4-Synthese-Inhibitor Zileuton zeigte einzelne positive Effekte [24]. Die grundsätzliche Frage, ob rAAV-2-Vektoren eine Reexpression des funktionellen Gens für FALDH in defekten Zellen er- 
Tab. 1 Geeignete Genodermatosen für rAAV-2-vermittelte Gentherapie [mod. n. 5]

\begin{tabular}{|c|c|c|}
\hline Genodermatose & Gendefekt & $\begin{array}{l}\text { Funktionale } \\
\text { cDNS-Länge (Nukleotide) }\end{array}$ \\
\hline x-chromosomale Ichthyose & Steroidsulfatase & 1550 \\
\hline Lamelläre Ichthyose & Transglutaminase 1 & 2450 \\
\hline Sjögren-Larsson Syndrom & $\begin{array}{l}\text { Fettaldehyddehydro- } \\
\text { genase }\end{array}$ & 1550 \\
\hline $\begin{array}{l}\text { Junktionale Epidermolysis } \\
\text { bullosa }\end{array}$ & $\begin{array}{l}\text { Laminin } 5-\gamma 2 \text { Kette } \\
(\text { LAMC2) }\end{array}$ & 1900 (verkürzt) \\
\hline Ehlers-Danlos Typ VI & Lysylhydroxylase 1 & 2100 \\
\hline Refsum Syndrom & $\begin{array}{l}\text { Phytanoyl-CoA Hydro- } \\
\text { xylase }\end{array}$ & 1050 \\
\hline Morbus Gaucher & Glukozerebrosidase & 1650 \\
\hline $\begin{array}{l}\text { Papillon-Lefevre/Haim- } \\
\text { Munk Syndrom }\end{array}$ & Cathepsin C & 1900 \\
\hline Albinismus Typ I & Tyrosinase & 1600 \\
\hline $\begin{array}{l}\text { Kongenitale erythro- } \\
\text { poetische Porphyrie }\end{array}$ & $\begin{array}{l}\text { Uroporphyrinogen III } \\
\text { Synthase }\end{array}$ & 1300 \\
\hline Xeroderma pigmentosum & $X P-A,-B,-C,-D$ & $910,2400,2500,2400$ \\
\hline
\end{tabular}

lauben, wurde bereits in einer FALDH-mutierten Hamsterzelllinie bearbeitet. Dort konnte die FALDH-Aktivität von ursprünglich unter $10 \%$ der Norm nach Gentransfer der funktionellen FALDH-Sequenzen in defekten Zellen auf normales Niveau angehoben werden [25]. Vorläufige Daten an Keratinozyten von SLSPatienten bestätigen eine Anhebung zumindest auf das Niveau von heterozygoten Merkmalsträgern, die phänotypisch unauffällig sind. Im Rahmen dieser Versuche scheinen rAAV-2-Vektoren das Potenzial zur genetischen Wiederherstellungstherapie bei SLS zu haben. Ungeklärt bleibt jedoch, bei SLS wie auch bei allen weiteren Genodermatosen, welcher Applikationsweg der viel versprechendste ist. Ist es beispielsweise möglich, genetisch modizifierte Keratinozytenstammzellen dauerhaft zu transplantieren, und wenn ja, wieviel Hautoberfläche muss transplantiert werden, bis sich ein therapeutischer Nutzen einstellt? Oder wird ausschließlich ein direkter In-vivo-Gentransfer hilfreich sein? Würde der Gentransfer über die Haut auch zu einer systemischen Verteilung von FALDH führen und könnten dadurch die neurologischen und systemischen Defizite bei SLS abgemildert werden ?

Die letzte Frage zieht das Konzept mit ein, die Haut als „Biorektor“ zur Therapie von systemischen Enzym-Mangelerkrankungen nutzen zu wollen [26]. Hierbei würde ein Gen, dessen Genprodukt systemisch reduziert ist, in der Haut überexprimiert, von dort sezerniert und über die Blutzirkulation verteilt. Die prinzipielle Funktionalität dieses Konzeptes konnte bereits in einzelnen Tiermodellen beispielsweise zur Therapie der Hämophilie aufgezeigt werden, jedoch noch nicht unter Verwendung von rAAV-2-Vektoren.

Zusammenfassend lässt sich feststellen, dass rAAV-2 durchaus als Vektor zur Hautgentherapie geeignet erscheint. In den entsprechenden Modellen konnten sowohl Effekte zur Förderung der Wundheilung als auch zur Genrekonstitution von Genodermatosen am Beispiel von SLS erzielt werden. Obwohl die Forschungstätigkeit nach wie vor auf die Überprüfung der prinzi- piellen Funktionalität ausgerichtet und modellhaft ist, motivieren die Ergebnisse zur Weiterentwicklung der Konzepte. Unabhängig von der Wahl des Vektorsystems müssen jedoch in den kommenden Jahren speziell zur Gentherapie von Genodermatosen weitere grundlegende Fragestellungen bearbeitet werden, wie das Problem der Immunantwort auf Vektor oder Genprodukt, die gezielte Integration von Fremd-DNS an spezifischen Stellen im Chromosom zur Vermeidung einer insertionellen Mutagenese, der gezielte Gentransfer in Keratinozytenstammzellen in vivo oder die Entwicklung geeigneter Transplantationsverfahren zur dauerhaften Akzeptanz ex vivo-modifizierter Stammzellen im Epidermis- bzw. Haarfollikelverbund.

\section{Literatur}

${ }^{1}$ Braun-Falco M, Hallek M. Hautgentherapie-Perspektiven des Gentransfers in Keratinozyten. Hautarzt 1998; 49: 536-544

${ }^{2}$ Braun-Falco M. Gentherapeutische Konzepte zur Förderung der Wundheilung. Hautarzt 2002; 53: 238-243

${ }^{3}$ Khavari PA, Rollman O, Vahlquist A. Cutaneous gene transfer for skin and systemic diseases. J Intern Med 2002; 252: 1 - 10

${ }^{4}$ Watt FM. Epidermal stem cells as targets for gene transfer. Hum Gene Ther 2000; 11: $2261-2266$

${ }^{5}$ Stilwell JL, Samulski RJ. Adeno-associated virus vectors for therapeutic gene transfer. Biotechniques 2003; 34: 148-150

${ }^{6}$ Büning H, Braun-Falco M, Hallek M. Progress in the use of adeno-associated viral vectors for gene therapy. Cells Tis Org 2004; 177: 139-150

${ }^{7}$ Hallek M, Girod A, Braun-Falco M, Wendtner CM, Bogedain C, Hörer M. Recombinant adeno-associated virus vectors. Cur Res Mol Ther 1998; 1: $417-430$

${ }^{8}$ Snyder RO, Flotte TR. Production of clinical-grade recombinant adenoassociated virus vectors. Curr Opin Biotech 2002; 13: 418-423

${ }^{9}$ Xiao X, Li J, Samulski RJ. Production of high-titer recombinant adenoassociated virus vectors in the absence of helper adenovirus. J Virol 1998; 72: $2224-2232$

${ }^{10}$ Dong J-Y, Fan P-D, Frizzell RA. Quantitative analysis of the packaging capacity of recombinant adeno-associated virus. Hum Gene Ther 1996; 7: $1201-1212$

${ }^{11}$ Braun-Falco M, Doenecke A, Smola H, Hallek M. Efficient gene transfer into human keratinocytes with recombinant adeno-associated virus vectors. Gene Ther 1999; 6: 432 - 441

${ }^{12}$ Hengge UR, Mirmohammadsadegh A. Adeno-associated virus expresses transgenes in hair follicles and epidermis. Mol Ther 2000; 2: 188 194

${ }^{13}$ Braun-Falco M, Hallek M. Recombinant adeno-associated virus (rAAV) vector-mediated cotransduction of CD70 and CD80 into human malignant melanoma cells results in an additive T-cell response. Arch Dermatol Res 2001; 293: $12-17$

${ }^{14}$ Douar AM, Poulard K, Stockholm D, Danos O. Intracellular trafficking of adeno-associated virus vectors: routing to the late endosomal compartment and proteasome degradation. J Virol 2001; 75: 1824-1833

15 Duan D, Yue Y, Yan Z, Yang J, Engelhardt JF. Endosomal processing limits gene transfer to polarized airway epithelia by adeno-associated viurs. J Clin Invest 2000; 105: 1573-1587

${ }^{16}$ Braun-Falco M, Eisenried A, Büning H, Ring J. Optimization of recombinant adeno-associated virus-mediated gene transfer in human keratinocytes. Arch Dermatol Res; eingereicht

17 Mah C, Qing KY, Khuntirat B, Ponnazhagan S, Wang X-S, Kube DM, Yoder MC, Srivastava A. Adeno-associated virus type 2-mediated gene transfer: role of epidermal growth factor receptor protein tyrosine kinase in transgene expression. J Virol 1998; 72: 9835-9843

${ }^{18}$ Deodato B, Arsic N, Zentilin L, Galeano M, Santoro D, Torre V, Altavilla D, Valdembri D, Bussolino F, Squadrito F, Giacca M. Recombinant AAV vector endocing human VEGF165 enhances wound healing. Gene Ther 2002; 9: $777-785$

${ }^{19}$ Galeano M, Deodato B, Altavilla D, Cucinotta D, Arsic N, Marini H, Torre V, Giacca M, Squadrito F. Adeno-associated viral vector-mediated human vascular endothelial growth factor gene transfer stimulates angiogenesis and wound healing in the genetically diabetic mouse. Diabetologia 2003; 46: 546-555 
${ }^{20}$ Galeano M, Deodato B, Altavilla D, Squadrito G, Seminara P, Marini H, Stagno d'Alcontres F, Colonna M, Calo M, Lo Cascio P, Torre V, Giacca $\mathrm{M}$, Venuti FS, Squadrito F. Effect of recombinant adeno-associated virus vector-mediated vascular endothelial growth factor gene transfer on wound healing after burn injury. Crit Care Med 2003; 31: 1017 1025

${ }^{21}$ Barrandon Y, Green H. Three clonal types of keratinocytes with different capacities for multiplication. Proc Natl Acad Sci USA 1987; 84: $2302-2306$

22 Möhrenschlager M, Rizzo WB, Kraus CS, Limbrock J, Cohen M, AntonLambrecht I, Abeck D, Ring J. Sjögren-Larsson Syndrom. Hautarzt 2000; $51: 250-255$

${ }^{23}$ Rizzo WB, Lin Z, Carney G. Fatty aldehyde dehydrogenase: genomic structur, expression and mutation analysis in Sjögren-Larsson syndrome. Chem Biol Interact 2001; 130-132: 297-307

${ }^{24}$ Willemsen MA, Lutt MA, Steijlen PM, Cruysberg JR, van der Graaf M, Nijhuis-van der Sanden MW, Pasman JW, Mayatepek E, Rotteveel JJ. Clinical and biochemical effects of zileuton in patients with the Sjögren-Larsson syndrome. Eur J Pediatr 2001; 160: 711 - 717

${ }^{25}$ Haug St, Ring J, Braun-Falco M. Adeno-associated virus vectors are able to restore fatty aldehyde dehydrogenase-deficiency in vitro. Implications for gene therapy in Sjögren-Larsson syndrome. Arch Dermatol Res; eingereicht

${ }^{26}$ Cao T, Wang X-J, Roop DR. Regulated cutaneous gene delivery: The skin as a bioreactor. Hum Gene Ther 2000; 11: 2297-2300

\section{Buchbesprechung}

Die Geschichte der Dermatologie an der Charité und in Berlin K. Harnack, W. Lehnert, E. D. Schulze, H. J. Tietz, H. Winter

Berlin: BMV, 2004. Kart. 25,45€. ISBN 3-88040-208-6

Ein Jahrzehnt nach der Wende ist es Zeit, die gemeinsamen und die gesonderten Wege unseres Faches zu reflektieren und zu verknüpfen. Geschichte ist ein Teil unserer Identität. Lehren aus derselben sind ein hohes Gut.

Klaus Harnack bemüht sich mit seinen Mitarbeitern um unser Fach Dermatologie und Venerologie und um dessen Einrichtungen in Berlin von 1710 - 1999 und legt ein umfassendes, einsichtsreiches, interessantes, ja faszinierendes Buch vor, das uns durch Zeit und Probleme führt. Geschmückt ist es mit vielen kleinen Portraits, mit Plänen und Abbildungen, ausgestattet mit manchen Biographien und gestaltet mit kritischer Liebe. Die Autoren verdienen Glückwünsche und unseren Dank.

Gedenktage der Dermatologie nannten es unsere Altvorderen, wenn sie besonders Erinnerungswertes festhielten, und manche Sternstunde war mit dabei. Hier werden wir mitgenommen zur Gründung als „Lazareth-Hauses“ 1710 und zur ersten Benennung als Charité 1727, zur Gründung der Berliner Universität 1810 mit dem Philosophen Johann Gottlieb Fichte als erstem Rektor, und zur Gründung der Hautklinik 1858.

Bemerkenswert ist auch, dass 1884 die Hautklinik von der Syphilisklinik getrennt wurde, eine Entwicklung, die sich im angelsächsischen Raum und in vielen Ländern bis heute gehalten hat, und dass es Edmund Lesser, dem ersten Berliner Ordinarius unseres Faches, 1902 gelang, die beiden Kliniken wieder zu vereinigen.

Die „hohe Zeit der Ernte“ der klinischen und experimentellen Dermatologie und der Venerologie vor hundert Jahren wird plastisch und in voller Breite dargestellt, wie auch die bauliche Entwicklung.

Bemerkenswert ist aber auch die Rückständigkeit im Ausbau und die Knappheit der Mittel, welche dazu führten, dass der Zürcher Ordinarius Bruno Bloch 1931 den eigentlich ehrenvollen Ruf an die Charité ablehnte. Die gigantischen Neubaupläne der NS-Zeit wurden nicht realisiert.

Nach 1945 war die Charité im Osten Berlins eingeengt und im Westen hat zunächst die Dermatologie am Rudolf-VirchowKrankenhaus unter Günter Stüttgen und dann auch diejenige am Benjamin Franklin Klinikum der FU unter Constantin Orfanos die Führung übernommen und weltweite Ausstrahlung erwirkt. Als Sternstunde wird die erste gemeinsame Sitzung der Dermatologen aus Ost und West der Stadt am 18.10.1989 unter Führung von Gisela Albrecht geschildert, die zum Zusammenschluss 1991 führte. Damit vertritt die 1886 gegründete Berliner Dermatologische Gesellschaft (BDG) unser Fach in der Hauptstadt wiederum mit Festigkeit und Konstanz, was von besonderer Bedeutung ist, da die Umgestaltung der akademischen und sozialpolitischen Landschaft viel Unruhe bringt. Das Buch endet 1999 und seither ist noch viel geschehen.

Eine faszinierende Geschichte sehr gut dargebracht!

E. G. Jung, Heidelberg 